\title{
Ogilvie's Syndrome Presented as Angina: A Case Report
}

\author{
Zakariya Abdulazeez, Uyen Tran \\ Department of Acute Medicine, Medway Maritime Hospital, Kent, UK
}

Doi: 10.12890/2020_001517 - European Journal of Case Reports in Internal Medicine - ๔ EFIM 2020

Received: 23/01/2020

Accepted: 24/01/2020

Published: $18 / 05 / 2020$

How to cite this article: Abdulazeez Z, Tran U. Ogilvie's syndrome presented as angina: a case report. EJCRIM 2020;7: doi:10.12890/2020_001517.

Conflicts of Interests: The Authors declare that there are no competing interests.

This article is licensed under a Commons Attribution Non-Commercial 4.0 License

\section{ABSTRACT}

Ogilvie's syndrome is a non-mechanical, acute pseudo-obstruction of the colon, causing massive colonic dilation. Medical or surgical conditions can predispose patients to Ogilvie's syndrome; however, the pathogenesis and clinical findings are still not well understood. Here, we present a case of a 48-year-old male patient who presented to the Emergency Department with intermittent self-resolved left-sided lower chest pain on a background of ischaemic heart disease and positive risk factors for acute coronary syndrome. Troponin testing was negative and an electrocardiogram showed no acute changes. Chest radiography showed a dilated bowel under the left hemidiaphragm and a computed tomography (CT) scan of the abdomen-pelvis confirmed the diagnosis of Ogilvie's syndrome. The patient was treated conservatively with a short period of nil by mouth and intravenous fluids.

\section{LEARNING POINTS}

- Non-cardiac causes of chest pain should be always considered even in patients with previous cardiac history, especially those patients for whom there is no evidence to support recurrent cardiac ischaemia.

- Acute colonic pseudo-obstruction (Ogilvie's syndrome) can be presented as chest pain that mimics angina pectoris.

- Chest radiography is of great value in cases of acute chest pain; a dilated bowel segment can be the only finding of Ogilvie's syndrome in the initial assessment.

\section{KEYWORDS}

Chest pain, acute coronary syndrome, Ogilvie's syndrome, pseudo-obstruction of the colon

\section{CASE DESCRIPTION}

A 48-year-old male presented to the Emergency Department with a 2-hour history of intermittent left-sided lower chest pain, described as heaviness on the lower chest radiating to the upper abdomen. The intensity of the pain varied from 8/10 to 4/10 on the pain scale, associated with some shortness of breath. The patient reported experiencing similar discomfort intermittently over the previous few weeks before this particular presentation. He was known to have asthma, hypercholesterolaemia and a history of myocardial infarction 3 years earlier, which was treated with coronary angioplasty and stenting. The patient had been admitted to the hospital 10 times in the previous 2 years with similar symptoms and treated for angina given his cardiac history, despite unchanged electrocardiograms (ECGs), negative troponin testing and no evidence of recurrent ischaemia on follow-up cardiac perfusion scans. He had a repeated coronary angiogram a year before this presentation, which showed stable coronary artery disease. Constipation was also reported by the patient during this episode but he was able to pass flatus. The recording of vital signs showed blood pressure of $104 / 65 \mathrm{mmHg}$ and a pulse rate of $61 \mathrm{bpm}$. Chest examination was unremarkable with a clear chest and normal heart sounds on auscultation. Abdominal examination revealed a soft non-tender abdomen with mild distension. Laboratory blood tests were grossly normal with negative troponin and D-dimer results. The ECG showed sinus rhythm (Fig. 1), with no acute changes compared to previous ECGs. Chest radiography showed an elevated left hemidiaphragm and dilation of the colon at the splenic flexure (Fig. 2). For this reason, an abdominal radiograph was requested, which revealed extensively dilated large bowel loops (Fig. 3). A computed tomography (CT) scan of the abdomen-pelvis was carried out (Fig. $4 A-C$ ), confirming a diagnosis of large bowel 
dilation and no mechanical obstruction. The cardiology and surgical teams at the hospital were consulted, and a myocardial perfusion scan confirmed previous myocardial infarction but no significant myocardial ischaemia. On this basis, and by excluding the possible differential diagnoses, a diagnosis of Ogilvie's syndrome was made. The patient was treated conservatively with a short period of nil by mouth and intravenous fluids. His antianginal medications were reviewed.

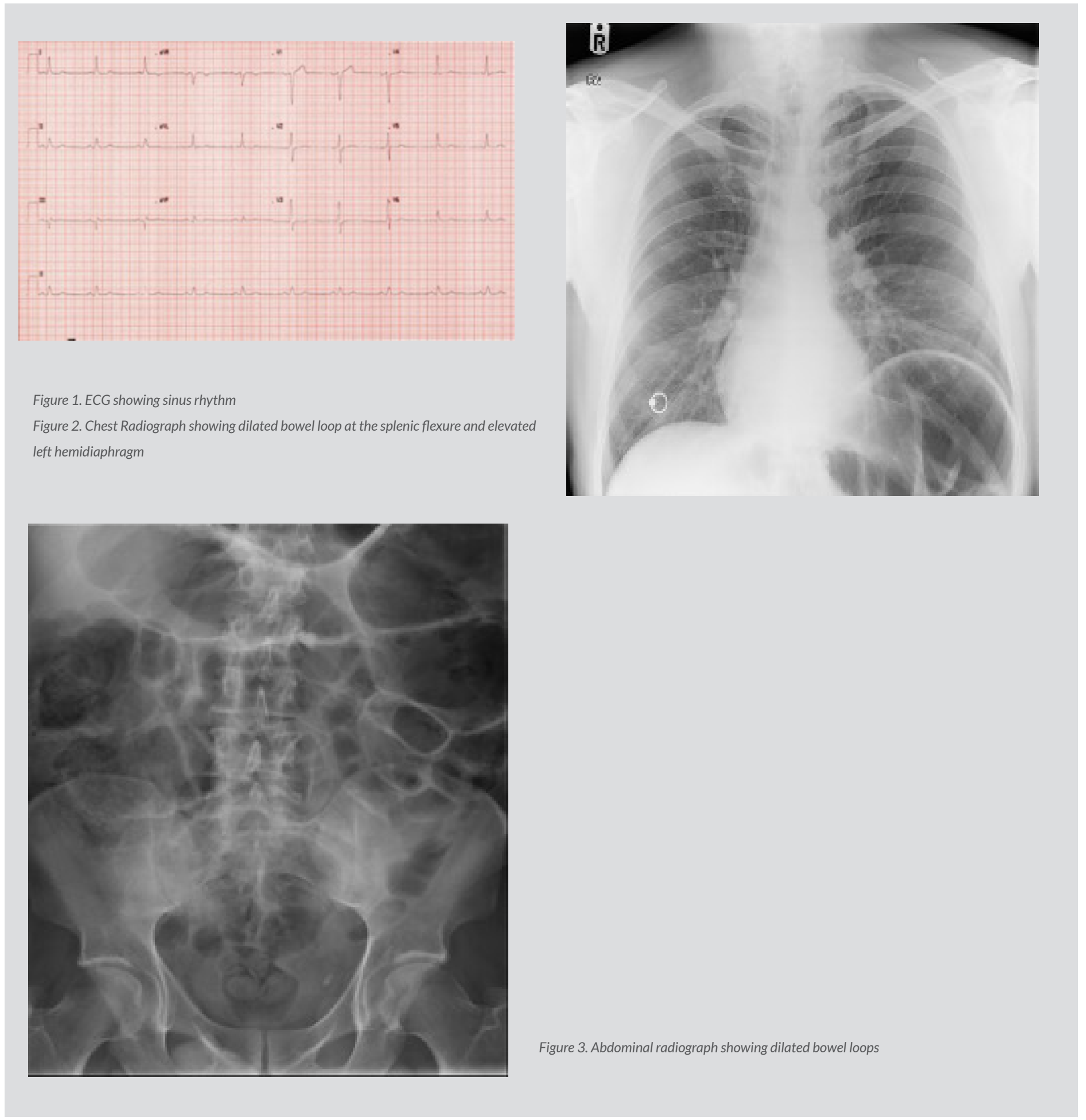



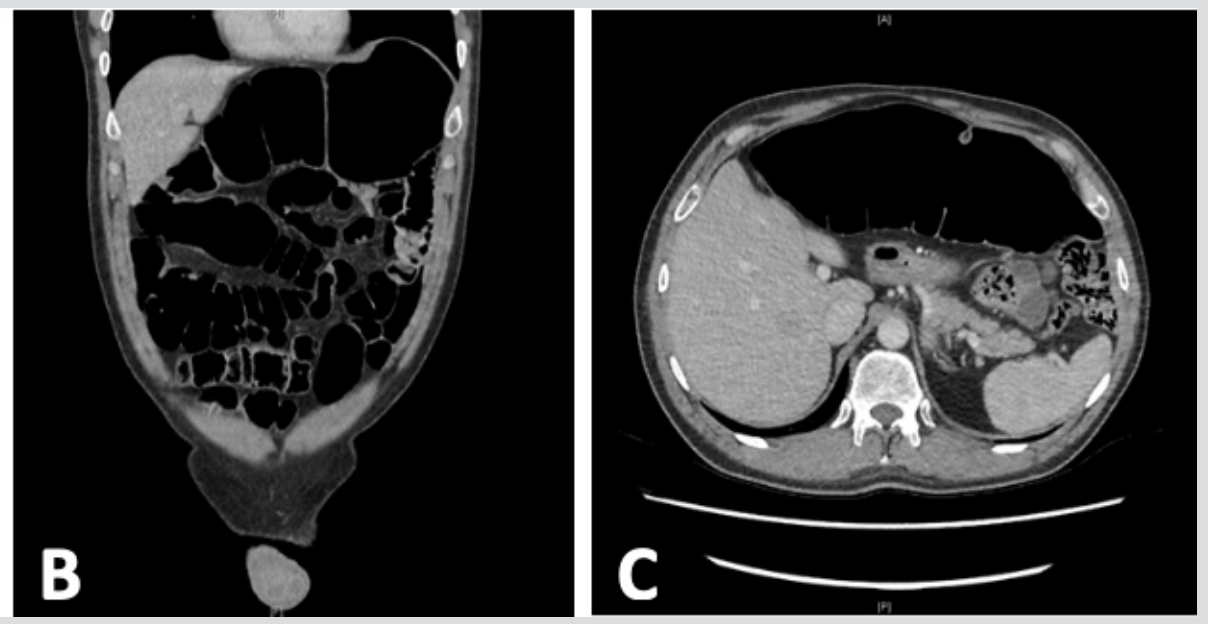

Figure 4. Computed tomography (CT) scan of abdomen-pelvis showing dilated large bowels

\section{DISCUSSION}

Ogilvie's syndrome is a non-mechanical, acute pseudo-obstruction of the colon, causing massive colonic dilation with common symptoms of nausea, vomiting, abdominal distension, abdominal pain and altered bowel motions. The pathogenesis and clinical findings of Ogilvie's syndrome are still not well understood. Some medical and surgical conditions can predispose patients to Ogilvie's syndrome, such as those reported in debilitated individuals ${ }^{[1,2]}$. Furthermore, the clinical suspicion of a diagnosis of Ogilvie's syndrome is usually confirmed by abdominal radiography and a CT scan ${ }^{[2]}$. In the current case presentation, the finding of a dilated bowel loop segment on the chest radiograph was the hint that further investigations should be carried out, thereby establishing the diagnosis of Ogilvie's syndrome.

Chest pain/angina pectoris is one of the most common presenting complaints in the Emergency Department. Although studies show that non-cardiac chest pain is very common, the initial evaluation should always exclude life-threatening causes. The wide range of differential diagnoses for chest pain can be challenging when assessing patients with an acute attack of chest pain ${ }^{[3]}$. An example of a challenging chest pain scenario is angina pectoris in patients with a normal coronary angiogram ${ }^{[4]}$. Cardiac troponins are sensitive and specific serum markers of myocardial cell injury, but they can also be elevated without cardiac injury ${ }^{[5]}$.

Acute coronary syndrome with electrocardiographic changes and troponin elevation caused by bowel obstruction has been reported in rare cases ${ }^{[6,7]}$. In 1 case report, an adult female presented with epigastric pain and ST elevation ECG changes attributed to recurrent small bowel obstruction ${ }^{[6]}$. Other gastrointestinal pathologies, such as acute cholecystitis, have also been reported to cause ECG changes, alteration of the coronary blood supply and bradycardia, which can be attributed to stimulation of the vagus nerve ${ }^{[5,8]}$.

In conclusion, non-cardiac causes of chest pain should always be considered even in patients with previous cardiac history, especially those patients for whom there is no evidence to support recurrent cardiac ischaemia.

\section{REFERENCES}

1. Maloney N, Vargas HD. Acute intestinal pseudo-obstruction (Ogilvie's syndrome). Clin Colon Rectal Surg 2005;18(2):96-101.

2. Pereira P, Djeudji F, Leduc P, Fanget F, Barth X. Ogilvie's syndrome - acute colonic pseudo-obstruction. J Visc Surg 2015;152:99-105.

3. Campbell KA, Madva EN, Villegas AC, Beale EE, Beach SR, Wasfy JH, et al. Non-cardiac chest pain: a review for the consultation-liaison psychiatrist. Psychosomatics 2017;58(3):252-265.

4. Yilmaz A, Sechtem U. Angina pectoris in patients with normal coronary angiograms: current pathophysiological concepts and therapeutic options. Heart 2012;98:1020-1029.

5. Demarchi MS, Regusci L, Fasolini F. Electrocardiographic changes and false-positive troponin I in a patient with acute cholecystitis. Case Rep Gastroenterol 2012;6:410-414.

6. Patel K, Chang NL, Shulik O, DePasquale J, Shamoon F. Small bowel obstruction mimicking acute ST-elevation myocardial infarction. Case Rep Surg 2015;2015:739147.

7. Parikh M, Amor MM, Verma I, Osofsky J, Paladugu M. Small bowel obstruction masquerading as acute ST elevation myocardial infarction. Case Rep Cardiol 2015;2015:685039.

8. Ozeki M, Takeda Y, Morita H, Miyamura M, Sohmiya K, Hoshiga M, et al. Acute cholecystitis mimicking or accompanying cardiovascular disease among Japanese patients hospitalized in a Cardiology Department. BMC Res Notes 2015;8:805. 